\title{
INTERPRETING THE FIELD EVIDENCE OF PAST ICE SHEETS: STRUCTURAL STABILITY AND GENERICITY
}

\author{
by
}

J.F. Nye

(H.H. Wills Physics Laboratory, Tyndall Avenue, Bristol BS8 1TL, U.K.)

\section{ABSTRACT}

Ice sheets are always advancing or retreating, and these processes are not uniform along the ice edge. The result is that lines of maximum and minimum extent are envelopes of successive instantaneous ice edges. Generically, the envelopes can possess only two kinds of special points: crossings and cusps. These will of ten be arranged in characteristic patterns (unfoldings of higher mathematical singularities). The recognition of these features makes possible a novel approach to the interpretation of field evidence. It is shown, for example, how separate families of parallel erosional and depositional features seen at an interior site are related by coming together in lines of cusps at the envelopes, which may be marked by terminal moraines.

\section{STRUCTURAL STABILITY AND GENERICITY}

The mathematical notions of structural stability and genericity (Thom, 1975, Arnold, 1983) can be used fruitfully (even though naively) in geophysics, and in glaciology in particular. The behaviour of the edge of an ice sheet provides a good illustration.

\section{THE EDGE OF AN ICE SHEET}

A real ice sheet is never in a steady state. As it waxes and wanes its outline advances and retreats, but the process will not occur exactly in unison all around the edge. This would be particularly marked in a continent-sized ice sheet which spanned a wide range of latitudes. For example, a maximum advance would not be reached at all points around the edge simultaneously. Exactly synchronous behaviour at the ice edge would occur only under very special conditions, such as one might find in a mathematical model, but never in nature. The model would represent a non-generic (special) case, to be contrasted with the generic (non-special) case always presented in nature. Alternatively expressed, the mathematical model would be structurally unstable, because the slightest perturbation could upset the synchronous behaviour of the ice edge.

Our task will be to look for features involving the motion of the ice edge that are structurally stable - that is to say, features that survive perturbation - because these are the features that we should expect to find in nature. Of course, this is merely one example of the use of the principle of structural stability. In general, the principle tells us when our models are too special (non-generic) and when they are general enough. It tells us to seek the generic case.

\section{THE HISTORY SURFACE AND ITS PROJECTION}

Suppose now we take map coordinates $x, y$ and at a given time $T$ draw the outline of the ice sheet. Repeat this for all times and then stack the outlines one above the other to produce a surface, the history surface, in the space
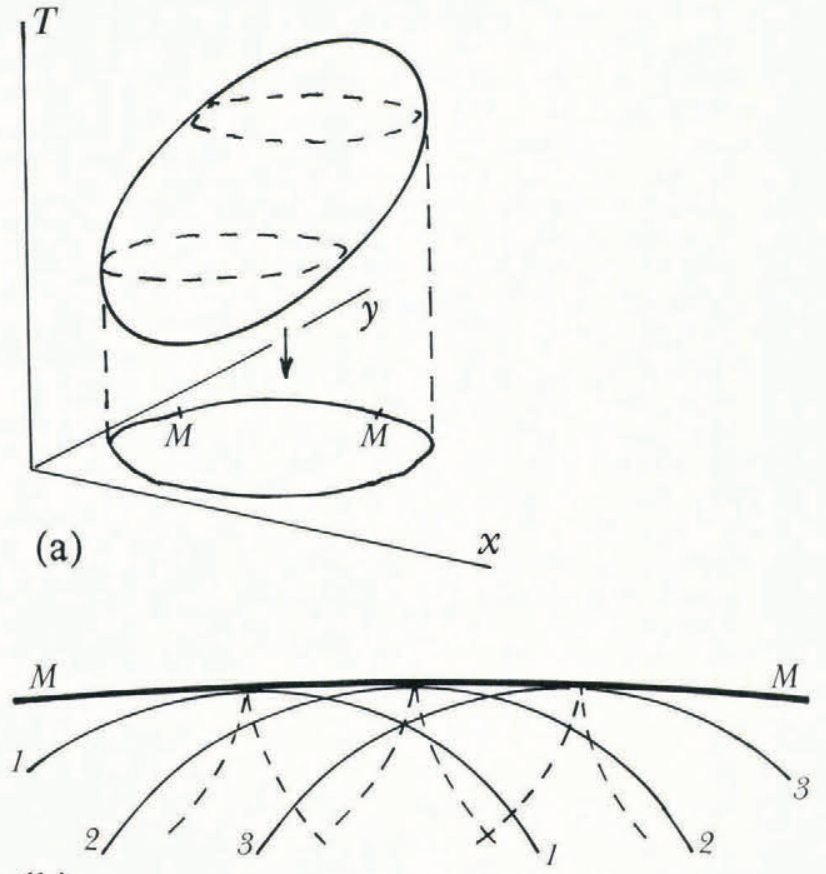

(b)

Fig. 1(a). The history surface in $(x, y, T)$ space projects down to give a fold line in the $(x, y)$ plane. (b). A portion MM of the fold line in the $(x, y)$ plane. Successive positions of the ice edge are marked $1,2,3$. $\mathrm{MM}$ is the line of maximum advance, an envelope, which may be a terminal moraine. The ice sheet does not reach MM at all points simultaneously. The broken lines indicate flow directions (see section 4).

( $x, y, T$ ) with the $T$ axis vertical (Fig. 1a). Its equation may be written $f(x, y, T)=0$; the instantaneous ice outlines are the sections $T=$ constant.

In reality the ice edge (suitably defined) may not be smooth and will certainly possess detail on a variety of scales. However, one is free to select a scale of spatial smoothing suited to whatever map scale is chosen. One can also select a suitable smoothing over time - for example, averaging over one or more years. Then the history surface will indeed be smooth. This is a vital property that we shall need in a moment. The history surface will be closed at top and bottom (the end and the beginning of the ice sheet), but it will have bulges and hollows, and possibly more complicated features as well. Two examples of sections through history surfaces parallel to the $T$ axis, derived from observations, appear in the paper by Boulton and others (1985, Figs. 13 and 14). Now suppose, viewing the surface along the $T$ direction, we project it down on to the $x, y$ plane. The curve we obtain, where the surface folds under itself, represents the line of maximum advance of the ice 


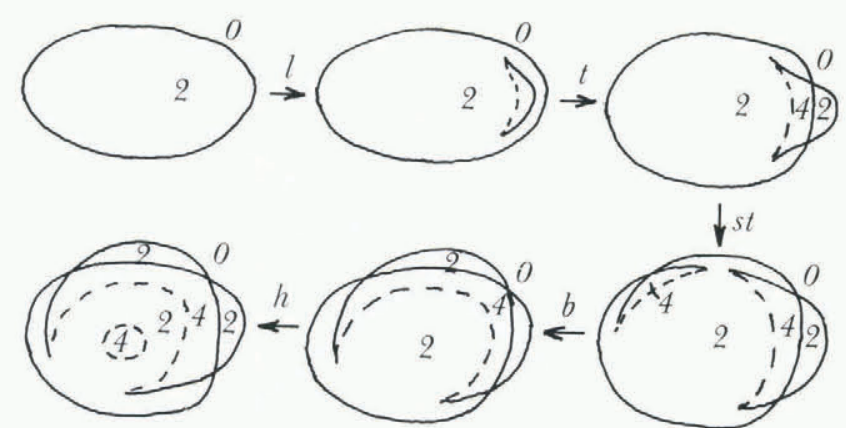

Fig. 2. Any one of these maps in the $x, y$ plane could represent the history of an ice sheet. The full curves are envelopes denoting local maxima of advance; the broken curves are envelopes denoting local minima. They join in cusps. To pass from one scenario to another requires lips (1), tangency (t), swallowtail (st), beak-to-beak (b) or hole (h). The numbers show the multiplicity.

sheet, and it will in fact be the envelope of the outlines we started with (Fig. 1b). It will not follow any one of these outlines exactly, even along a portion of its length, for that would mean that the ice reached a maximum of advance simultaneously all along part of its edge, which is non-generic. Thus, if the maximum advance is marked by a terminal moraine, this follows an envelope and its date varies along its length.

There will also be places where the ice sheet reached a local minimum and, once again, these will lie on an envelope, this time corresponding to hollows or constrictions in the history surface. The rule is that, if the tangent to the history surface parallel to the $T$-axis lies outside the enclosed solid, there is a local maximum of advance; if inside, there is a local minimum. In fact the envelope of the instantaneous outlines of the ice sheet consists of one or more continuous curves. Some theoretical examples of envelopes are shown in Figure 2, which we shall return to later. The curves can cross one another, and they contain special cusp points where the envelope changes from denoting a maximum of advance to denoting a minimum. Because the history surface is smooth, by construction, cusps and crossings are the only kinds of special points that genericity allows (Arnold, 1986). Anything more special would be structurally unstable and will not be found in nature.

Figure 3 a shows a cusp in more detail. On the line $\mathrm{PP}^{\prime}$ the ice edge successively advances, retreats, advances and retreats; on a curve of $x: T$ there are two maxima separated by a minimum. On $\mathrm{QQ}^{\prime}$ the first maximum has merged with the minimum, to leave merely a horizontal inflexion; while along $\mathrm{RR}^{\prime}$ there is just a single maximum of advance. Figure 3b may help the reader to visualise the situation.

With the coordinate system shown in Figure $3 a$ the history surface at the cusp has the local form: $f(x, y$, $T)=T^{3}-a y T+b x=0$. The line on the surface which gives the projected cusp is given by $f=0, \partial f / \partial T=0$, which yields $x \propto \mathrm{T}^{3}, y \propto T^{2}$. Thus in the $x, y$ plane the projected cusp has the standard form $x \propto y^{3 / 2}$. Notice that $\partial T / \partial y$ becomes infinite at $C$. Thus, if there were a terminal moraine along the upper (maximum) arm of the cusp, its date $T$ would change infinitely fast as one approached $C$. C marks a place where the ice edge lingered for an unusually long time before readvancing. An interesting feature is that each cusp has associated with it both a characteristic length and a characteristic time: the length is $b^{2} / a^{3}$ and the time is $b / a$.

Whereas the ice edge has traversed most of the field twice, once while advancing and once while retreating, it has traversed the area within the cusp four times. We shall call this number the multiplicity. We can say that the envelope lines separate areas of different multiplicity, and the multiplicity changes by \pm 2 as one crosses any envelope line.

At a crossing of envelope lines (Fig. 4) two maxima (or minima) of advance separated in time happen to have
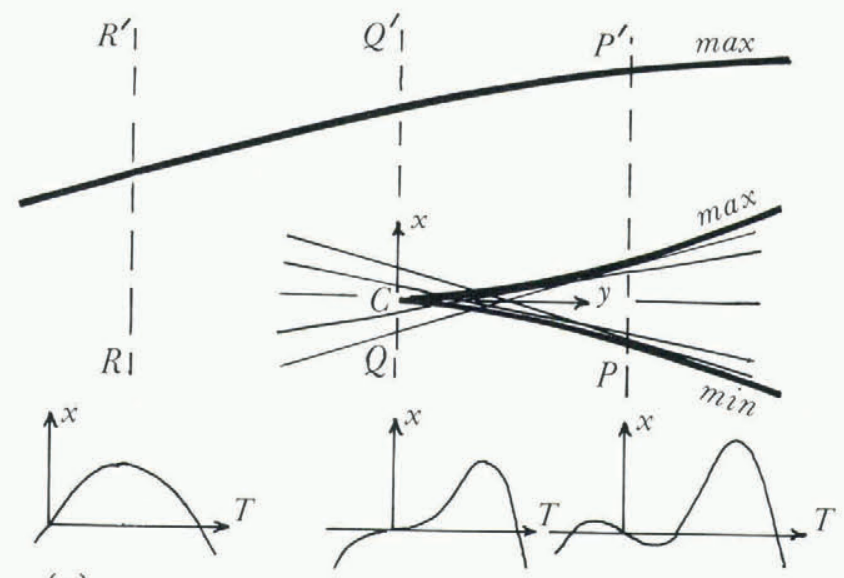

(a)

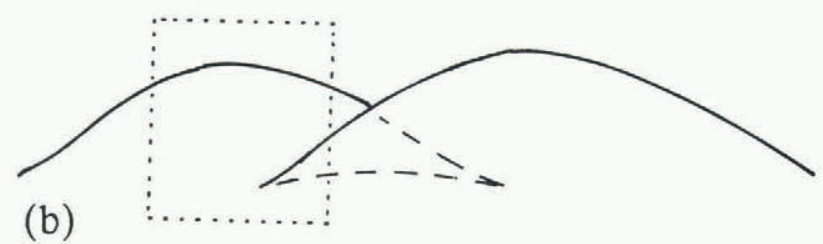

Fig. 3(a). A cusp in the envelope in the $x, y$ plane. Thin lines indicate successive ice-edge positions that envelope the cusp (they are drawn locally straight purely for simplicity). The upper uncusped envelope denotes a separate maximum. The lower diagrams show the movement of the ice edge at three different locations. (b). First interpret this diagram as showing two hills in a landscape, one partly behind the other. The broken lines are the extra portions of horizon that would be seen if the nearer hill were transparent. Now identify the contents of the rectangular box with the envelopes in (a) We are looking along the $T$ axis. The whole horizon is an unfolded swallowtail singularity.

occurred at the same place. If both maxima formed terminal moraines, an envelope crossing would be a point where the date of the most advanced moraine abruptly changed.

The successive diagrams in Figure 2 show how one can pass conceptually in a continuous way from one arrangement of envelopes (one history) to another - as, for example, by continuously varying some parameter $\lambda$ in a model of the process. $\lambda$ could be, in principle, almost any significant quantity appearing in the model, like, for example, the power in the Glen flow law, or a parameter controlling the roughness of the bed. A particularly interesting choice for $\lambda$ would be a parameter controlling the amount of smoothing used to construct the history surface from the field data. Thus, in this case, altering $\lambda$ would amount to altering the

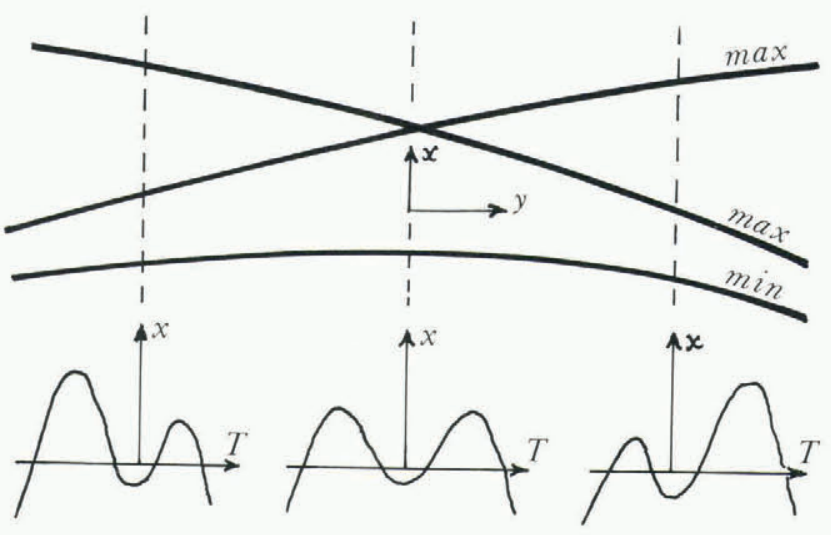

Fig. 4. A crossing of envelopes in the $x, y$ plane and the corresponding movement of the ice edge. Compare the central part of Figure $3 \mathrm{~b}$. 
degree of resolution that one wished to use in viewing the data. The various generic events, such as lips, occur as the resolution is changed. For a single parameter the generic events illustrated in Figure 2 are lips (birth of two cusps), tangency (of two folds at different heights), swallowtail, beak-to-beak (merging of two cusps), and hole (appearance of a hole in the enclosed solid). The last one would represent, for example, the oasis in an ice sheet produced by a temporary hot spot on the rock bed.

It is useful to call the volume enclosed by the history surface the history solid. Then other generic events, not illustrated, obtained by varying one parameter would be as follows: a cusp in the envelope crosses a fold originating at a different height; a history solid appears or disappears as an ellipsoid at a point (the reverse of the hole); two separate history solids, or voids in a history solid, join or disconnect at a point as (locally) a double cone. These last events give different projected envelopes according to whether the $T$-axis lies inside or outside the cone. It is much easier to draw the projected envelopes in the generic case than the families of instantaneous ice edges.

The usefulness of thinking in this way, introducing an adjustable parameter to pass from one history to another, is that the single fixed arrangement of envelopes actually realised can then be seen as an unfolding (an opening out) of higher singularities (Thom, 1975). The highest singularity, which in the simplest case is a point in the threedimensional space $(x, y, \lambda)$, plays the role of an organizing centre, introducing order into the two-dimensional sections in $(x, y)$ space.

\section{FAMILIES OF FLOW DIRECTIONS}

What are the implications of these ideas for the geomorphological traces of flow direction in past ice sheets? Lineation patterns, formed by features such as drumlins, fluted ground moraine and striations, do not reflect the ice-sheet-wide pattern of flow at any one time (e.g. Boulton and others, 1985). If deposition and erosion occurred uniformly under an ice sheet there would be a continuous range of flow directions at each place. New lineations will tend to overprint old ones, and those finally exposed will predominantly reflect the latest movement pattern. Nevertheless, there are many instances where several distinct flow directions are visible at the same place, so that traces of old flow directions can certainly survive. Moreover, there are theoretical arguments (Boulton, 1987: 37) to suggest that both deposition and, to a lesser extent, erosion are greatest towards the edge of an ice sheet. Therefore, it may be helpful to consider a precise simplified model in which lineation patterns reflect flow direction only at the moving ice edge; this can then be used as a basis for arguing about more complicated models.

Construct, therefore, families of flow directions, which will usually be roughly perpendicular to the instantaneous ice edge (this assumes approximately level ground - the sides of an outlet glacier would be exceptions). At any place there will be $0,2,4 \ldots$ sets of flow directions, one for each advance and one for each retreat. The number of sets will equal the multiplicity. At an envelope (Fig. 1b) two sets of flow directions run together in a continuous line of cusps (standard cusps of the form, on suitable coordinates, $x \propto y^{3 / 2}$ ). Figure 5 illustrates, for example, how four families of striations labelled $\alpha, \beta, \gamma, \delta$ are related in a region where there is an unfolded swallowtail. Each section of the envelope marks the merging of two out of the four families. Notice that $\alpha$ and $\gamma$ become the same family on the extreme right, as do $\beta$ and $\delta$ on the extreme left.

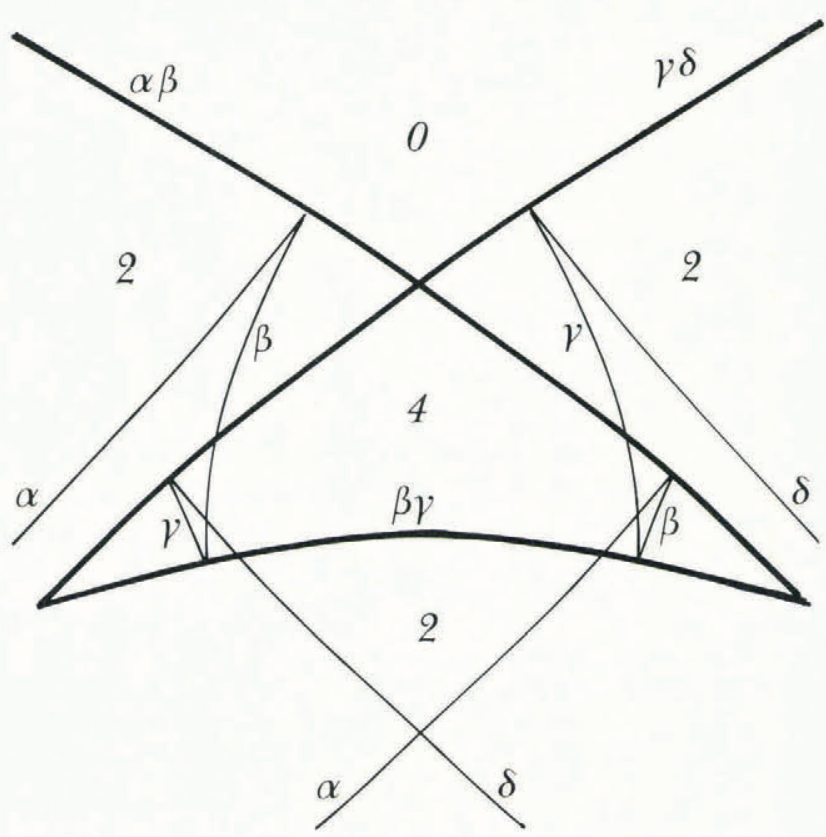

Fig. 5. The relation between four families of flow direction lines $\alpha, \beta, \gamma, \delta$ at an unfolded swallowtail in the envelope (thick line). This refers to a model where flow direction is significant only at the moving ice edge. Only two representative lines from each family are drawn. The numbers are the number of families in each area, the multiplicity.

We see from this example how different families of flow directions observed at a given site have a specific relationship when continued to the envelope. This knowledge, and the recognition of the cusped nature of the envelope, provide a theoretical framework to help in the interpretation of the of ten confusing field evidence of ice movement in past ice sheets.

\section{ACKNOWLEDGEMENTS}

I am grateful to Dr J.H. Hannay, Dr M.E.R. Walford and the referees for valuable comments.

\section{REFERENCES}

Arnold, V.I. 1983. Geometrical methods in the theory of ordinary differential equations. Berlin, Springer-Verlag, Chapter 3.

Arnold, V.I. 1986. Catastrophe theory. Second edition. Berlin, Springer-Verlag, Chapter 12.

Boulton, G.S. 1987. A theory of drumlin formation by subglacial sediment deformation. In Menzies, J. and J. Rose, eds. Drumlin Symposium. Proceedings of the Drumlin Symposium/First International Conference on Geomorphology/Manchester/16-18 September 1985. Rotterdam, A.A. Balkema, 1987, 25-80.

Boulton, G.S., G.D. Smith, A.S. Jones, and J. Newsome, 1985. Glacial geology and glaciology of the last mid-latitude ice sheets. J. Geol. Soc., London, 142(3), 447-474.

Thom, R. 1975. Structural stability and morphogenesis. Reading, MA, Benjamin. 